\title{
O Teste de Fotos de Profissões (BBT) de Achtnich: histórico e pesquisas desenvolvidas no Brasil
}

\author{
Sonia Regina Pasian - Universidade de São Paulo ${ }^{1}$ \\ Erika Tiemi Kato Okino - Universidade de São Paulo \\ Lucy Leal Melo-Silva - Universidade de São Paulo
}

\begin{abstract}
Resumo
Este trabalho descreveu o histórico de desenvolvimento e as principais características técnicas do Teste de Fotos de Profissões (BBT), sistematizando as investigações científicas desenvolvidas no Brasil com este instrumento projetivo de clarificação das inclinações motivacionais. Este teste foi criado na Suíça, por Martin Achtnich, na década de 70, sendo introduzido no Brasil em 1982 por André Jacquemin, que adaptou o BBT para o contexto sociocultural brasileiro, elaborando o BBT-Br nas versões masculina e feminina. Foram apresentadas as pesquisas brasileiras que utilizaram esse teste, com diferentes objetivos e grupos etários, demonstrando sua adequada validade e utilidade como instrumento auxiliar nos processos de orientação vocacional/profissional, seleção de pessoal e reorientação de carreira. A presente revisão crítica da literatura nacional sobre o BBT procurou oferecer a seus utilizadores suporte teórico abrangente e estimulador de reflexão e de pesquisas com essa técnica.

Palavras-chave: BBT; Avaliação psicológica; Orientação profissional/vocacional; Técnica projetiva; Interesses.
\end{abstract}

\section{The Achtnich's Berufsbilder-Test (BBT): history and researches in Brazil}

\begin{abstract}
This paper described the history and the principal characteristics of the Berufsbilder Test (BBT), systematizing the scientific researches developed in Brazil with this projective technique of interests clarification. This test was created in Switzerland by Martin Achtnich in the 1970's and was introduced in Brazil in 1982 by André Jacquemin, who adapted it to the Brazilian social-cultural context. He developed the BBT-Br in both the masculine and the feminine version. This paper also presented the Brazilian researches that used it with their different objectives and age groups, demonstrating its validity and utility as a helpful instrument in the vocational guidance process, personal selection and career reorientation. The present critical review of national literature about the BBT aimed at offering theoretical support for its users and to stimulate considerations and new researches with this technique.

Keywords: BBT; Psychological assessment; Vocational guidance; Projective technique; Interests.
\end{abstract}

\section{Orientação profissional/vocacional e avaliação psicológica}

Os recursos da avaliação psicológica, embora úteis e promissores nos processos de orientação profissional/ vocacional, são ainda foco de polêmica na realidade brasileira, como bem apontaram Jacquemin, Melo-Silva e Pasian (2002). Os múltiplos questionamentos sobre os instrumentos de exame psicológico em geral e, em especial em suas aplicações na área de orientação profissional/ vocacional, segundo Draime e Jacquemin (1989), ocorreram em função do longo período em que foram utilizados pelos psicólogos sem a preocupação com estudos de sua validade e/ou sua padronização ao contexto sociocultural brasileiro, sem mencionar a inadequação em suas utilizações. Ressaltaram, portanto, a importância dos estudos de padronização e validação das técnicas a serem aplicadas e a qualificação do profissional que fará uso das mesmas.

Apesar de não existir consenso entre os orientadores profissionais sobre a utilização de técnicas de avaliação psicológica, há uma concordância geral sobre a necessidade de um correto diagnóstico das vivências das pessoas que procuram por orientação profissional/ vocacional para possibilitar uma intervenção adequada e assertiva sobre a problemática. Barros (2005) afirma que a maioria dos profissionais que realizam orientação profissional/vocacional concorda que o diagnóstico psicológico se constitui como uma importante etapa nesse processo. Essa etapa diagnóstica seria a norteadora na escolha das

${ }^{1}$ Endereço para correspondência:

Universidade de São Paulo - Faculdade de Filosofia, Ciências e Letras de Ribeirão Preto - Depart. de Psicologia e Educação

Centro de Pesquisas em Psicodiagnóstico

Avenida Bandeirantes, 3.900 - Monte Alegre - Ribeirão Preto-SP 
estratégias a serem utilizadas, decorrentes da compreensão alcançada sobre o indivíduo a ser atendido, tendo como foco a resolução de sua problemática de definição ocupacional.

Por sua vez, Melo-Silva e Jacquemin (2001b) discutiram a relevância e os limites dos processos psicodiagnósticos dentro da orientação profissional, enfatizando a necessidade de se utilizar instrumentos tecnicamente qualificados aos propósitos desse tipo de intervenção. Essa idéia também foi reforçada por Savickas (2004), ao afirmar que a avaliação psicológica possibilita, ao psicólogo, acesso a dados mais objetivos sobre a dinâmica vivenciada pelo cliente, inserido em seu ambiente social.

Dentre as várias possibilidades de avaliação psicológica do indivíduo na área de orientação profissional/ vocacional em nosso país, o Conselho Federal de Psicologia, tendo por base a meta de aprimoramento dos instrumentos psicológicos (CFP, 2003), emitiu parecer favorável ao Teste de Fotos de Profissões - BBT. Destaque também foi oferecido a essa técnica de avaliação psicológica por Abade (2005), ao afirmar que o BBT está entre os dois testes projetivos estudados no Brasil na área de orientação profissional, porém é o único com parecer favorável do CFP e, portanto, utilizado nas práticas.

As possibilidades informativas do instrumento BBT para clarificação das inclinações motivacionais do indivíduo oferecem um lugar de relevância para essa técnica no campo da orientação profissional/vocacional (MeloSilva \& Jacquemin, 2001a). Pensando-se nessas implicações, o presente trabalho almeja apresentar um histórico dessa técnica de avaliação psicológica, sua origem e seus fundamentos, bem como relatar os estudos científicos que a fundamentam, desde sua criação até a atualidade, com ênfase nos trabalhos desenvolvidos no Brasil.

Dessa forma, este trabalho justifica-se na medida em que o conhecimento científico sobre o BBT encontrase esparso e, muitas vezes, restrito a seus pesquisadores, dificultando a possibilidade de fundamentação do uso desse método em nosso país. Assim, considera-se que o presente estudo, ao discriminar e descrever detalhadamente os trabalhos de pesquisa desenvolvidos no Brasil com o BBT, ou seja, sua revisão bibliográfica em nosso contexto, poderá vir a contribuir como um estímulo a novas investigações com esse método, otimizando suas chances de aprimoramento técnico-científico, como almejado por todos os utilizadores de instrumentos de avaliação psicológica.

Para tanto, foram consultados os estudos científicos elaborados com o BBT disponíveis em manuais da técnica, dissertações e teses, bem como em livros, periódicos científicos, livros de resumos e anais de congressos científicos da área de orientação profissional/vocacional ou de avaliação psicológica desde a década de 70 (época de criação do BBT) até a atualidade, focalizando a análise sobre os materiais publicados no contexto brasileiro. Para essa busca dos estudos científicos com o BBT tomou-se por base o acervo bibliográfico sobre o tema disponibilizado pelo pesquisador André Jacquemin, responsável pela introdução dessa técnica projetiva no Brasil, ampliando-o de tal forma a tentar retratar, no presente trabalho, um mapeamento da produção científica nacional sobre o BBT, além de breve histórico de seu processo de criação e de desenvolvimento científico.

\section{O BBT: origem e fundamentos}

O Teste de Fotos de Profissões ou Berufsbilder-Test (BBT) é uma técnica projetiva de avaliação psicológica e foi elaborado por Martin Achtnich na década de 1970 (Achtnich, 1971, 1973). Tem se destacado como recurso útil e válido na investigação das variáveis significativas envolvidas no processo de escolha ocupacional, ou seja, na avaliação dinâmica dos interesses (Achtnich, 1979, 1991). Trata-se de um instrumento composto por 96 fotos de profissionais no exercício de sua profissão, apresentando-se nas versões masculina e feminina. Este teste foi introduzido no Brasil na década de 1980 e almeja avaliar as tendências motivacionais do indivíduo, resultando em informações sobre suas estruturas primária e secundária de interesses e de rejeições de atividades, podendo ser aplicado com eficiência em processos de orientação profissional (Jacquemin \& Pasian, 1991; Leitão, 1993).

No processo de elaboração do BBT, Achtnich (1991) recorreu a princípios da combinação de fatores proposta pela Teoria de Personalidade de Szondi (1970). Este, por sua vez, havia elaborado um conjunto de formulações próprias sobre os componentes da personalidade e suas ligações com os interesses pessoais, compondo variáveis representativas de estruturas de inclinação motivacional, interferentes em toda a vida dos indivíduos e, portanto, também em suas escolhas ocupacionais. Com base nesses princípios e após longo processo de investigações empíricas, Achtnich elaborou o seu Teste de Fotos de Profissões (Berufbbilder Test), conhecido como BBT. Nessa técnica projetiva, as estruturas de inclinação motivacional do indivíduo poderiam ser investigadas por meio de escolhas e de rejeições de atividades, de ambientes, de instrumentos de trabalho, apresentados em fotografias de indivíduos em situação de trabalho. Achtnich (1991) apresentou oito fatores (chamados radicais de inclinação) como os elementos básicos para se classificar as tendências, as aspirações fundamentais e as inclinações essenciais dos 
interesses e da vida, assumindo-os como fatores de determinação (embora não exclusivos, por reconhecer a influência sociocultural e a realidade imediata) das escolhas humanas, inclusive no processo de decisão profissional. De forma sintética, esses oito fatores componentes da personalidade e das motivações humanas seriam: fator $\mathrm{W}$ (estar em contato com o outro, colocar-se à disposição; revela sensibilidade e subjetividade); fator $\mathrm{K}$ (utilização da força física, da agressividade, da perseverança); fator $\mathrm{S}$ (senso social, dividido em duas vertentes inter-relacionadas: $\mathrm{S}_{\mathrm{H}}$ - ajuda e $\mathrm{S}_{\mathrm{E}}$ - disposição); fator $\mathrm{Z}$ (mostrar, estar em evidência, ser valorizado, representar; valorizar o esteticamente belo); fator $\mathrm{G}$ (imaginação criativa, intuição e idéias); fator $\mathrm{V}$ (objetividade, conhecimento, organização, racionalidade e precisão); fator $\mathrm{M}$ (lidar com a matéria, com limpeza e com fatos passados); fator $\mathrm{O}$ (oralidade, dividida em duas vertentes: $\mathrm{O}_{\mathrm{N}}$ - nutrição e $\mathrm{O}_{\mathrm{R}}$ - comunicação).

A estrutura de inclinação motivacional do indivíduo seria constituída a partir da combinação desses fatores entre si. Achtnich (1991) afirmava que nenhuma atividade profissional poderia ser caracterizada somente por um dos fatores acima apresentados, concebendo-as como multifatoriais. Dessa forma, as fotos que compõem o BBT também foram estruturadas de modo a representar pelo menos uma estrutura bifatorial, a saber: um fator primário (representando a atividade principal em foco e apresentado em letra maiúscula) e um fator secundário (representando o objeto profissional e os instrumentos da atividade em foco, apresentado em letra minúscula). Dessa forma, por exemplo, a foto 41 do BBT, na versão masculina, seria tecnicamente proposta como representativa do fator principal W e do fator secundário g, ou seja, seria uma foto $\mathrm{Wg}$, ilustrada na foto do florista (profissional em ação).

No BBT de Achtnich (1991), a estrutura de inclinação profissional seria elaborada a partir da análise da classificação das 96 fotos do teste em três grupos distintos: fotos de preferência (que agradam o indivíduo), fotos rejeitadas (que desagradam) e fotos indiferentes. Essa escolha das fotos seria dirigida não apenas pelos aspectos racionais de suas representações, mas o aplicador deveria enfatizar ao respondente a questão de que sua classificação dos estímulos deveria passar pelas impressões afetivas sobre as fotos. Com isso seria possível avaliar o processo implícito nas decisões dos indivíduos e, portanto, também em sua escolha profissional, fornecendo informações sobre a organização de suas preferências e rejeições em relação a uma diversidade de possibilidades representadas nas fotos. O BBT também prevê que o respondente descreva suas impressões sobre as fotos escolhidas (associações), com o objetivo de acessar as reflexões que o indivíduo realizou sobre suas escolhas e suas interpretações sobre as mesmas, constituindo-se no caráter projetivo dessa técnica, onde as peculiaridades interpretativas têm lugar e podem ser Psico-USF, v. 12, n. 2, p. 173-187, jul./ dez. 2007 devidamente exploradas e, posteriormente, analisadas no conjunto das evidências técnicas obtidas.

Achtnich (1991) elaborou o seu instrumento de avaliação das inclinações motivacionais (BBT) reconhecendo, por sua ampla prática em atendimentos clínicos, a estreita ligação entre a satisfação das necessidades do indivíduo e seu sucesso profissional e pessoal, resultado da integração das características de personalidade e dos interesses. A fundamentação teórica do BBT pressupõe que os fatores de inclinação motivacional constituem necessidades que podem ser satisfeitas no exercício da profissão (embora não exclusivamente), de forma que elas se organizam na busca por gratificação na realidade. Aponta ainda que essa gratificação básica da estrutura de inclinações motivacionais do indivíduo, em atividades laborais ou cotidianas, seria um elemento favorecedor de sua saúde mental, representando um aspecto de preservação da própria vida.

\section{O impacto do BBT em orientação profissional/vocacional}

A partir da criação do BBT, na década de 70, na Suíça, muitos outros estudiosos passaram a reconhecer nessa técnica uma alternativa promissora para se conhecer características motivacionais e de personalidade dos indivíduos, passando a ser utilizado como recurso técnico em vários serviços de orientação profissional/vocacional na Europa. Na década de 80 o BBT já havia sido incorporado em programas de orientação profissional/vocacional de adolescentes na Suíça e na Bélgica, posteriormente em Portugal e em outros países europeus, embora poucas publicações científicas tenham se efetivado a respeito dessas aplicações do método projetivo, sobretudo pelo caráter clínico desses serviços de atendimento a adolescentes. Apesar disso, o impacto do BBT na Europa foi significativo, como atestam estudos de diferentes centros de pesquisa (Achtnich, 1988; Boudrenghien, 1983; Chaghaghi \& Jobin, 1983; De Ketele, 1982; Foulon, 1981; Leitão, 1984, 1993; Leleux, 1982; Lievyns, 1987; Marchand, 1983; Walgraffe, 1989).

No processo de utilização do BBT na prática em orientação profissional/vocacional, uma preocupação básica norteava os profissionais interessados nesse método projetivo: esta técnica seria adequada a adolescentes de um ambiente sociocultural diferente daquele onde foi criado o instrumento, no caso, do contexto suíço-alemão? Essa questão norteou, portanto, trabalhos de investigação inicial da validade do BBT em diferentes países, dirigidos fundamentalmente à verificação da forma de percepção e de captação/elaboração das fotos por parte dos adolescentes, verificando sua adequação ao poder de representação dos oito fatores da inclinação motivacional propostos por Achtnich (1991). 
Com esse objetivo, portanto, de verificar a validade do Teste de Fotos de Profissões (BBT) de Achtnich, algumas pesquisas foram realizadas ainda na década de 80. Destaca-se, a título de exemplo desse processo, dos vários trabalhos existentes, o estudo desenvolvido por Foulon (1981) na Bélgica e por Leitão (1984; 1993) em Portugal.

As possibilidades informativas do BBT logo fizeram com que esse instrumento projetivo de clarificação das inclinações motivacionais chegasse também ao Brasil, em 1982, ou seja, praticamente na mesma época em que houve grande disseminação do método na Europa, num artigo científico publicado nos Arquivos Brasileiros de Psicologia. Nas palavras de seu autor:

Esta comunicação visa divulgar um novo instrumento de avaliação da estrutura de interesses, o Teste de Achtnich, inspirado nas teorias de Szondi e ponto central do processo de orientação no Centre de Consultation et d'Orientation Psychologique et Pédagogique da Université Catholique de Louvain, Bélgica. A originalidade do material, a facilidade de sua aplicação, assim como o dinamismo que se evidencia na relação psicólogo-orientando, quando de sua utilização, são aqui salientados. (Jacquemin, 1982, p. 127)

A partir dessa introdução do BBT no Brasil, variadas possibilidades de sua aplicação tiveram início neste país. Investimentos científicos de verificação de suas características técnicas foram realizados, bem como inúmeras aplicações clínicas do instrumento em diferentes centros de orientação e de reorientação profissional, fortalecendo a riqueza do método. Diante dessa realidade, o presente trabalho tem por objetivo apresentar o histórico do BBT no Brasil, bem como seus passos de desenvolvimento até a atualidade, descrevendo as pesquisas realizadas com o instrumento.

\section{Pesquisas com o BBT no Brasil}

Como referido anteriormente, a introdução do Teste de Fotos de Profissões (Berufsbilder Test) de Martin Achnitch, o BBT, ocorreu em 1982, por André Jacquemin, docente da Faculdade de Filosofia, Ciências e Letras de Ribeirão Preto da Universidade de São Paulo. Na época emergia como uma "instrumentação diferenciada para completar ou até substituir as técnicas tradicionais de avaliação de interesses" (Jacquemin, 1982, p. 131). Essa alternativa metodológica auxiliar para os processos de orientação profissional/vocacional, sem dúvida, estimulou sua introdução em serviços aplicados de atendimento a adolescentes, embora, na proposta original de Achtnich, poderia ser aplicado em processos de necessidade de mudança profissional, de seleção de pessoal ou em análises de fracassos ocupacionais em adultos. Esses diferentes usos do BBT aos poucos foram se concretizando no contexto nacional, pautados por várias investigações científicas sobre este método projetivo, considerando o alerta inicial do próprio introdutor do teste no Brasil:

É certo que o teste de Achtnich precisa ainda de muitas pesquisas para se tornar um verdadeiro instrumento de medida. É necessário também obter resultados sobre grupos profissionais mais amplos e diversificados para se determinar melhor os fatores primários e secundários a eles ligados, verificar a validade interna do teste, isto é, o grau de concordância entre a atividade representada na foto e os fatores que a caracterizam, além de ser importante estudarse a validade do material para outras culturas que não a européia, como é o caso do Brasil. (Jacquemin, 1982, p. 131)

A seguir, passar-se á aos relatos dos trabalhos de investigação científica com o BBT desenvolvidos no Brasil, classificados de acordo com o objetivo, a amostra e/ou método utilizado. As pesquisas foram organizadas nas seguintes categorias: estudos de validade, pesquisas normativas (forma masculina e feminina), estudos de caso, estudos de processos de intervenção em orientação vocacional/profissional, aprimoramentos técnicos e estudos na área de recursos humanos.

\section{Estudos de validade}

Logo a partir das primeiras aplicações do BBT no contexto sociocultural brasileiro, alguns trabalhos apresentando essa técnica à comunidade científica foram debatidos no VI Congresso Lationamericano de Rorschach e Outras Técnicas Projetivas (Jacquemin, 1985). Durante o mesmo evento, Jacquemin, Xavier, Wierman e Lima (1985) apresentaram resultados de estudo sobre a validade interna na forma masculina do BBT, aplicada individualmente em 88 alunos de segundo e terceiro anos do ensino médio de Ribeirão Preto (SP), de ambos os sexos. Seus dados apontaram que as descrições elaboradas pelos adolescentes sobre as fotos do BBT masculino confirmaram os fatores Achtnich em praticamente metade dos estímulos, com tendência a essa confirmação nas demais fotos. Sugeriram, no entanto, a possibilidade de modificar o material original do BBT, de modo a adaptá-lo às características socioculturais do Brasil, sendo esse estudo posteriormente publicado por Jacquemin (1989). Ainda no mesmo evento científico acima referido, foi apresentado o estudo de Jacquemin e Nunes (1985), relatando os resultados da investigação sobre a validade interna da forma feminina BBT na realidade brasileira. Para tanto, avaliaram 40 estudantes do sexo feminino do ensino médio de Ribeirão Preto. Concluíram pela necessidade de revisar também essa 
versão feminina do BBT para a realidade sociocultural brasileira. Esse trabalho foi também apresentado, em 1990, num outro congresso científico internacional (Jacquemin \& Nunes, 1990).

$\mathrm{Na}$ seqüência dos trabalhos sobre o BBT no Centro de Pesquisas em Psicodiagnóstico da Faculdade de Filosofia, Ciências e Letras de Ribeirão Preto da Universidade de São Paulo, Nunes (1989) desenvolveu seu mestrado, sob orientação do professor André Jacquemin, investigando também a validade interna (de conteúdo) das formas masculina e feminina do teste. Além disso, procurou verificar especificamente a eficácia da forma feminina do BBT quanto a sua validade de conteúdo, comparando as possibilidades informativas com os dados da forma masculina, que inicialmente era empregada para ambos os sexos. Para tanto, trabalhou com 50 adolescentes do sexo feminino, matriculadas nos dois últimos anos do ensino médio (público e particular) de Ribeirão Preto, com idades variando entre 15 e 21 anos. Dentre essa amostra, 25 adolescentes foram avaliadas com a forma masculina e as outras 25 foram avaliadas com a forma feminina do BBT. Os dados obtidos possibilitaram a confirmação do fator primário em 66 fotos da versão masculina e em 70 fotos da versão feminina do teste. Esses resultados foram posteriormente publicados nos anais $\mathrm{da}$ conferência da International Association for Educational and Vocational Guidance (IAEVG, AIOSP, sigla em francês) (Jacquemin \& Nunes, 1995).

Acomodados os parâmetros técnicos básicos do BBT na realidade sociocultural brasileira, pensouse em investigar as evidências de validade desse instrumento em termos preditivos. Dessa forma, Bernardes (2000) desenvolveu seu mestrado objetivando verificar a possibilidade prognóstica do BBT em orientação profissional/vocacional, pesquisa que foi publicada mais tarde no formato de livro (Bernardes \& Jacquemin, 2002). Este foi um estudo longitudinal com o BBT, realizado com dez adolescentes (seis do sexo feminino e quatro do sexo masculino) do terceiro ano do ensino médio de Ribeirão Preto, com idades que variaram entre 16 e 20 anos no momento da primeira etapa da avaliação. A segunda etapa avaliativa ocorreu aproximadamente três anos mais tarde, momento no qual foram reaplicados o BBT, um questionário e uma entrevista clínica, com objetivo de visualizar relatos sobre história de vida e interesses ocupacionais. Os dados obtidos permitiram a verificação da eficiência do BBT como instrumento prognóstico de escolhas profissionais, além de possibilitar a análise do dinamismo das pulsões e das necessidades motiva- cionais dos indivíduos avaliados, evidenciando a flexibilidade intrínseca no processo de organização dos interesses e das motivações ao longo do período de vida examinado.

Um trabalho de pesquisa bastante interessante focalizou a investigação dos pressupostos de Achtnich (e do método projetivo BBT) de que a satisfação das necessidades motivacionais e dos interesses pessoais seria promotora de bons níveis de saúde psíquica e geral nos indivíduos. Trata-se de um trabalho de mestrado (Campos, 2003) que investigou a congruência/ incongruência entre o perfil vocacional (evidenciado pelo BBT) e o perfil da profissão desempenhada por adultos. Examinou, também, o índice de procura por serviços de saúde nesses indivíduos avaliados. Seus resultados foram consistentes em evidenciar que os indivíduos mais satisfeitos com sua ocupação apresentavam maior correspondência com suas necessidades motivacionais, conforme dados do BBT, além do que apresentavam poucas intercorrências em sua saúde geral. Esse estudo permitiu concluir que a incongruência entre necessidades/interesses pessoais e as atividades profissionais realizadas relacionam-se direta e positivamente com sintomas de doença. Conseguiu-se, dessa forma, clara evidência dos pressupostos teóricos elaborados por Achtnich para o BBT (Achnitch, 1991).

Ainda na perspectiva de demonstração empírica da utilidade e da validade clínica do BBT em orientação profissional/vocacional, Melo-Silva, Noce e Andrade (1999, 2003) estudaram os alcances terapêuticos de processos individuais e grupais de orientação de adolescentes, evidenciados por meio dos indicadores dessa técnica projetiva de avaliação psicológica. Para isso, as autoras analisaram a estrutura de inclinação profissional de 136 adolescentes (39 do sexo masculino e 97 do sexo feminino) com idade entre 16 e 20 anos, examinados em processos individuais ou grupais, sendo um dos instrumentos utilizados o BBT. Os dados foram avaliados quantitativa e qualitativamente e comparados com dados normativos brasileiros. As autoras apresentaram, discutiram e compararam os dados de produtividade e a estrutura de inclinação motivacional positiva e negativa, de acordo com o sexo dos sujeitos. Nas considerações finais, as autoras ressaltaram a importância do BBT no processo de orientação profissional/vocacional, tanto para o orientando como para o orientador. Por fim, destacaram semelhanças e diferenças na estrutura de interesses motivacionais e ocupacionais em virtude da variável sexo, a partir das informações obtidas com esta técnica projetiva.

Buscando aprimorar o conhecimento sobre os processos psicológicos inerentes ao desenvolvimento, especialmente no momento da escolha ocupacional durante a adolescência, Jardim-Maran (2004, 2007) recorreu 
aos recursos do BBT-Br e do Questionário Desiderativo como estratégias informativas, associados a um roteiro de entrevista semi-estruturado sobre história de vida. Avaliou, individualmente, por meio dessas técnicas, 60 estudantes entre 16 e 19 anos de idade, do terceiro ano do ensino médio de Ribeirão Preto, igualmente distribuídos quanto ao sexo e ao tipo de escola (pública x particular). Os resultados do BBT-Br e do Desiderativo foram examinados independentemente por psicólogos com experiência em avaliação psicológica, procurando-se oferecer fidedignidade ao processo analítico e comparativo dos seus respectivos indicadores técnicos. Foi possível evidenciar, por meio desse trabalho, sinais de interface qualitativa entre os fatores de inclinação mais escolhidos ou rejeitados no BBT-Br e os mecanismos de defesa/conteúdos rejeitados no Questionário Desiderativo. Os resultados apontaram ainda que os adolescentes indicaram vivenciar sentimentos de vulnerabilidade e de instabilidade pessoal diante da escolha profissional (alunos do terceiro ano do ensino médio), porém acompanhados por sinais de bons recursos adaptativos (defesas eficientes). Houve evidências de algumas diferenças significativas no processamento psicodinâmico dos adolescentes em relação ao sexo e à origem escolar (escola pública ou particular), sugerindo especificidades defensivas associadas ao treinamento adaptativo de vida experenciado por esses adolescentes em seu contexto sociocultural. Em termos gerais, a associação do Questionário Desiderativo ao BBT-Br mostrou-se um procedimento enriquecedor para a compreensão dos temores e das ansiedades pelos adolescentes neste período da vida, muitas delas bastante freqüentes e por eles compartilhadas. Uma das características gerais identificadas nesses adolescentes foi a alta prevalência da indecisão quanto às atividades profissionais satisfatórias ou não às suas demandas psíquicas, evidenciando reduzido conhecimento de si e da realidade ocupacional contemporânea.

\section{Estudos normativos: BBT masculino}

No ano de 1991, na conferencia da International Association for Educational and Vocational Guidance, da IAEVG/AIOSP em Lisboa, Jacquemin (1995) apresentou os resultados iniciais de padrões normativos do BBT no contexto brasileiro, por se ter verificado, em estudos anteriores de sua autoria e de seu grupo de pesquisa, influência relevante de fatores socioculturais no desempenho nessa técnica. Este trabalho apresentou o resultado da avaliação individual de 24 estudantes do sexo masculino dos dois últimos anos do ensino médio de Ribeirão Preto. Encontrou e comentou as importantes variações no padrão de escolhas das fotos do BBT entre os estudantes brasileiros e os suíços, apresentadas por Achtnich (1979), reforçando os argumentos sobre a necessidade de estudos específicos desse instrumento de avaliação psicológica em seus diferentes contextos de possível aplicação. Estas evidências foram também apontadas em outra publicação do grupo (Jacquemin e colaboradores, 1995).

Esses estudos brasileiros, com o objetivo de verificação da adequação do instrumento ao contexto nacional, verificaram que várias imagens das fotos não suscitavam as associações correspondentes aos respectivos fatores originalmente propostos por Achtnich (Jacquemin, 1995; Jacquemin e colaboradores, 1995). Esses resultados incentivaram a realização de outras pesquisas em busca da adaptação e padronização deste instrumento para o contexto sociocultural brasileiro. Primeiramente foi pesquisada a forma masculina do BBT (Jacquemin, 1997, 1999; Jacquemin e colaboradores, 1998). Este processo foi delineado em várias etapas, sendo a primeira para identificação das fotos que necessitariam de reformulação e a segunda etapa investigativa de escolha e de elaboração de fotos substitutas das originais (com problemas em sua validade), originando a criação do $\mathrm{BBT}$ masculino brasileiro, chamado de BBT-Br. A terceira parte dessa ampla investigação foi dirigida para a obtenção de padrões de resposta (normas) de desempenho de estudantes do ensino médio e universitário de Ribeirão Preto no BBT-Br. O detalhamento de cada um desses estudos está descrito a seguir.

Participaram, da primeira parte dessa ampla investigação, 91 adolescentes do sexo masculino, entre 15 e 19 anos, matriculados no segundo ou terceiro anos do ensino médio público e particular de Ribeirão Preto, submetidos individualmente ao BBT original (forma masculina). As associações elaboradas pelos respondentes sobre as fotos do BBT foram submetidas à avaliação de três juízes independentes, classificando-as de acordo com as categorias propostas por Achtnich para análise de cada estímulo de seu teste: profissional representado, função (atividade) representada, instrumento utilizado, local, objeto e objetivo da atividade profissional representada na foto, procurando-se identificar o fator motivacional ali representado (V, G, Z, W, K, M, O, S). Esse minucioso processo analítico permitiu a identificação de fotos que confirmavam e outras que não confirmavam (chamadas de "fotos problemáticas") o fator primário a elas atribuído por Achtnich (1991). O resultado dessa pesquisa concluiu pela necessidade de reformulação de 42 das 96 fotos originais do BBT masculino.

Deu-se início, então, à segunda parte da pesquisa de adaptação do BBT masculino ao contexto sociocultural brasileiro, também apresentada com minúcias em Jacquemin e colaboradores (1998). Foi a etapa de elaboração e de construção de novas fotos, representações mais adequadas para aqueles fatores Achtnich anteriormente não confirmados na percepção dos 
adolescentes brasileiros. Para representar os diferentes contextos de trabalho, vários profissionais (mínimo de dois por profissão) foram entrevistados em seus respectivos locais de trabalho para levantamento de informações sobre a sua prática de trabalho e definição das situações profissionais que melhor representassem aquele profissional na nova foto, ou seja, que melhor representasse o fator primário proposto por Achtnich. Pensando na qualidade do material fotográfico e na necessidade de adequada caracterização da profissão a ser representada, foram fotografadas várias situações reais de trabalho, registradas em diversos ângulos por um fotógrafo profissional, sob a orientação dos pesquisadores. Para cada uma das 42 profissões a serem representadas, o grupo de pesquisa selecionou, dentre os muitos estímulos retratados, aproximadamente sete fotos de cada atividade profissional estudada. Essas novas fotos foram submetidas a uma testagem com estudantes do ensino médio, entre 15 e 20 anos de idade, com o objetivo de avaliá-las quanto ao poder de representação do fator primário proposto por Achtnich (1991). As fotos foram apresentadas aos estudantes com o intuito de verificar o que estava sendo por eles percebido na foto, além de elegerem, entre as diferentes representações da mesma atividade, qual a melhor foto para representar cada uma das profissões investigadas. Para a definição da foto a substituir a original, a equipe de pesquisadores optou por privilegiar aquela que mais suscitou, nos sujeitos, verbalizações de funções ligadas ao fator primário proposto por Achtnich. Ao final desse longo processo adaptativo do BBT, surgiu, então, este novo conjunto de fotos, composto por 54 fotos originais e 42 reelaboradas, que passou a ser chamado de BBT-Br (forma masculina).

A terceira etapa desta pesquisa (Jacquemin, 1999; Jacquemin e colaboradores, 1998) teve por objetivo desenvolver normas avaliativas para o BBT-Br, focalizando-se estudantes do ensino médio e também grupos profissionais diversos. Com esse objetivo, foram avaliados, de forma coletiva, 472 alunos (224 da rede particular e 252 da rede pública) de ensino médio de Ribeirão Preto, além de 227 universitários da mesma cidade, sendo 69 estudantes da área de ciências exatas (química, engenharia civil e análise de sistemas), 76 da área de ciências humanas (jornalismo, direito e administração de empresas) e 82 da área de ciências biológicas (medicina, odontologia e biologia). Para além desses estudantes, foram avaliados, por meio de aplicação individual do BBT-Br, 31 profissionais (com, pelo menos, três anos de experiência em seu trabalho) dos nove cursos universitários anteriormente referidos, procurando-se caracterizar perfis de desempenho no teste em adultos em pleno exercício ocupacional, oferecendo, dessa forma, padrões analíticos adicionais para uso desta técnica de avaliação psicológica na Psico-USF, v. 12, n. 2, p. 173-187, jul./ de₹: 2007 realidade sociocultural brasileira contemporânea. Esse trabalho final acabou sendo publicado no formato de um manual do BBT-Br masculino, editado pelo CETEPP (Jacquemin, 2000) e também divulgado por Pasian (2007) no III Congresso Brasileiro de Avaliação Psicológica e XII Conferência Internacional de Avaliação Psicológica: Formas e Contextos, realizado em João Pessoa (Brasil).

\section{Estudos normativos: BBT feminino}

Posteriormente, em 1998, iniciou-se o trabalho de adaptação da forma feminina do BBT à realidade sociocultural brasileira. $\mathrm{O}$ estudo inicial desse processo teve por objetivo verificar o poder de representação dos oito fatores motivacionais de Achtnich nas fotos originais do BBT feminino. Nesse trabalho de Jacquemin e colaboradores (2000) concluiu-se pela necessidade de reformulação de 47 fotos das 96 originais desse teste. O trabalho desenvolvido a partir de então seguiu o mesmo procedimento adotado na forma masculina, acima relatado. $\mathrm{Ou}$ seja, inicialmente foram pesquisadas as atividades ocupacionais a serem representadas, depois os profissionais, voluntários da pesquisa, foram fotografados em seu ambiente real de trabalho. Esse amplo conjunto de fotos das atividades profissionais foi, então, submetido à análise de adolescentes, que avaliaram seu poder de representação dos diferentes fatores motivacionais de Achnitch, opinando sobre qual seria a melhor foto para representar cada uma das atividades propostas para compor o novo BBT. Desse trabalho resultou a composição da versão brasileira, adaptada a nosso contexto sociocultural, da forma feminina do BBT, chamada de BBT-Br feminino. Restava, por fim, o desenvolvimento de padrões normativos para a forma feminina do BBT-Br, o que foi desenvolvido a partir de uma amostra de 512 alunas do ensino médio de Ribeirão Preto, sendo 221 da rede particular e 291 da rede pública de ensino. Nesse processo de desenvolvimento de referenciais normativos do BBT-Br feminino também foram avaliadas 352 universitárias de Ribeirão Preto, de diferentes áreas científicas (111 da área de exatas, 135 de humanas e 106 de biológicas), representadas respectivamente por uma variedade de cursos universitários públicos e particulares. Esse longo processo de adaptação da forma feminina do Teste de Fotos de Profissões para o contexto cultural brasileiro foi publicado em vários trabalhos em eventos científicos e em revistas especializadas, representando as diferentes etapas do estudo, podendo ser encontrado em Jacquemin e colaboradores (2000, 2001, 2002, 2003), Okino e colaboradores (2003), Pasian (2007) e resultando no manual do BBT-Br feminino, publicado pelo CETEPP Jacquemin e colaboradores, 2006).

Paralelamente a esse longo processo de adaptação e de padronização do BBT à realidade sociocultural 
brasileira, outros pesquisadores desenvolveram estudos focalizando as possibilidades de aplicação desse instrumento de clarificação motivacional em diferentes contextos da orientação vocacional/profissional.

\section{Estudos de caso}

Procurando divulgar as possibilidades informativas do BBT para outros centros de pesquisa, Jacquemin e Nunes (1987) apresentaram um estudo de caso durante o XVI Congresso Internacional da Sociedade Internacional de Rorschach e Métodos Projetivos, realizado no Guarujá (SP, Brasil). Conseguiram, dessa forma, dar início a um intercâmbio científico relevante deste método projetivo com outros investigadores presentes ao evento, alcançando seus objetivos de divulgação do método.

Seguindo a perspectiva de investigação dos recursos investigativos e da aplicabilidade clínica do BBT, Melo-Silva e Santos $(1997,1998)$ e Melo-Silva e Jacquemin (2000), em dois estudos de caso, avaliaram adolescentes de 17 anos, do sexo masculino, cursando o $3^{\circ}$ ano do ensino médio. Para isso, fizeram uso de entrevista de triagem, questionário de vida confidencial, BBT e técnicas grupais, aplicados em 12 encontros de duas horas de duração. As narrativas desse estudo de caso foram submetidas à análise de conteúdo de duas histórias (a primeira aplicada no início e a segunda ao término do atendimento), elaboradas a partir das cinco fotos preferidas, que foram comparadas de acordo com as categorias: identificação dos personagens, capacidade de manejo do conflito profissional, desfecho (solução do conflito), sentimento de identidade e organização defensiva. Os autores foram unânimes na conclusão de que o procedimento complementar "história das cinco fotos preferidas" não substitui a aplicação convencional do BBT, mas constitui-se como um auxiliar favorecedor à clarificação do conflito vivenciado pelo orientando na definição de um projeto de vida, uma vez que o ajuda a desenvolver novas percepções sobre si e sua vida, tanto em termos pessoais como profissionais.

Em 1997, Sbardelini defendeu seu doutoramento em psicologia com uma tese que explorou as possibilidades do BBT na compreensão de casos de reopção de curso universitário e no estabelecimento de prognósticos da adaptação à nova escolha profissional, destacando o valor preditivo desse instrumento de avaliação psicológica. Participaram da primeira fase dessa pesquisa 299 estudantes que pediram reopção de curso na Universidade Federal do Paraná nos anos de 1993 e 1994, que responderam um questionário sobre suas necessidades e percurso de formação acadêmica e universitária até aquele momento. Já na segunda fase do estudo, 19 universitários foram selecionados (dentre os pertencentes ao grupo anterior) e avaliados individualmente mediante entrevista e BBT, a partir dos quais foram realizados estudos de caso. Dentre esses 19 sujeitos, dez eram do sexo masculino e nove do sexo feminino, com idades que variaram entre 21 e 36 anos. Quantitativamente, os dados obtidos possibilitaram a verificação dos seguintes resultados: a) predomínio de interesses variados nas mulheres, enquanto os homens indicaram sentimentos de indecisão quanto às escolhas; b) aspirações profissionais elevadas em todos os casos de reopção de curso avaliados; c) inclinações motivacionais dirigidas para atividades criativas e imaginativas no conjunto dos casos, envolvendo interesses por relações sociais de ajuda ao outro. Qualitativamente, os dados apontaram a necessidade de criação de um programa de esclarecimento, atendimento e orientação aos alunos ingressantes nos cursos universitários, com o objetivo de auxiliá-los no processo de autoconhecimento, integrando informações sobre os cursos escolhidos e o campo profissional dos mesmos. A autora destacou a riqueza das informações obtidas com o BBT, atestando, em seus estudos de caso, a relevância da contribuição desse instrumento projetivo para a compreensão da dinâmica psíquica dos processos de reopção profissional, sugerindo sua utilização nesses casos. Conseguiu, dessa forma, evidenciar a validade clínica do BBT em nosso contexto, trabalhando com universitários (Sbardelini, 1997).

Nessa mesma perspectiva, Welter (1998a) relatou um estudo de caso com uma adolescente, avaliada em dois momentos (aos 15 e aos 17 anos de idade, respectivamente) pelas duas formas do BBT: masculina (aplicada coletivamente) e feminina (aplicada individualmente). A autora apresentou e discutiu, com minúcia, as informações equivalentes dessas duas versões do teste nesse trabalho, identificando os mesmos fatores pulsionais nos dois momentos avaliativos da adolescente, além de ressaltar a importância da história das cinco fotos preferidas e das associações sobre as fotos como fatores relevantes para a validade das informações obtidas por meio do BBT. Essa evidência empírica foi de grande contribuição para o esclarecimento sobre a equivalência das duas versões do BBT quanto aos dados sobre as estruturas de inclinação de interesses dos indivíduos, apesar das diferenças implícitas nas fotos que compõem essas duas formas do teste.

Seguindo essa seqüência temática de apresentação dos trabalhos publicados no Brasil com o BBT, Jacquemin, Melo-Silva e Pasian (2002) e Melo-Silva e Noce (2004) apresentaram, no formato de capítulos de livros sobre orientação vocacional, estudos de caso com o Teste de Fotos de Profissões (BBT) enquanto método projetivo auxiliar nos processos implicados neste tipo de intervenção com adolescentes. Por meio das ilustrações clínicas das experiências de trabalho, conseguiram demonstrar a eficiência e a utilidade desse método projetivo no contexto da orientação vocacional/profissional no Brasil. Essas publicações são úteis na prática do orientador profissional 
tanto para avaliar as inclinações profissionais dos adolescentes quanto para avaliar o processo de intervenção.

\section{O BBT em processos de orientação vocacional/profissional}

Numa perspectiva de verificar as possibilidades de uso do BBT em processos de orientação profissional/ vocacional, Guelli e Jacquemin (1997) e Ribeiro (1998) confirmaram, empiricamente, a importância desse instrumento de avaliação psicológica em atendimentos clínicos de adolescentes, favorecendo seu autoconhecimento e estimulando o conhecimento da realidade profissional. Trata-se de um único estudo, publicado em duas etapas do trabalho, que teve por objetivo verificar possíveis mudanças nos adolescentes em um conjunto de variáveis implicadas no processo de escolha profissional, após realizarem o BBT e a técnica R-O. Para isso, os autores trabalharam com 30 adolescentes do sexo feminino que estavam cursando o segundo ano do ensino médio de Ribeirão Preto, com idades variando entre 16 e 17 anos e sem histórico de repetência escolar. Os resultados obtidos enfatizaram, sob o ponto de vista prático, a importância da utilização do R-O e do BBT como recursos de estimulação das adolescentes na preparação e na ampliação das informações sobre as opções e os próprios interesses profissionais, além de favorecer a apreensão das vinculações entre profissões e escolhas profissionais, em suas várias determinações.

$\mathrm{Na}$ perspectiva de avaliar diferentes possibilidades de intervenção grupal em orientação profissional/ vocacional, o trabalho de doutorado de Melo-Silva (2000), publicado posteriormente no formato de livro (Melo-Silva \& Jacquemin, 2001b), apresentou resultados de um estudo de aplicação do BBT como instrumento auxiliar na avaliação dessas intervenções terapêuticas. Estudaram uma amostra de 28 adolescentes do sexo feminino, matriculadas no segundo ano do ensino médio público de Ribeirão Preto, que foram divididas em quatro grupos operativos. Um desses grupos funcionou como controle dos demais, não sofrendo qualquer intervenção terapêutica, enquanto os três outros participaram de processos de orientação profissional/vocacional. Todos os estudantes foram avaliados, no início e no final do processo (após três meses), por meio do Questionário de Maturidade Profissional (QMP) e do BBT. A análise comparativa dos resultados desses dois momentos avaliativos entre os grupos evidenciou eficácia da intervenção realizada em orientação profissional/vocacional, atestando a importância desse tipo de processo com os adolescentes em momento de escolha ocupacional. Nessas avaliações das intervenções realizadas com as adolescentes, o instrumento projetivo BBT mostrou-se como valiosa técnica auxiliar aos processos de orientação profissional/vocacional, sendo recomendada sua utilização a partir dessas evidências Psico-USF, v. 12, n. 2, p. 173-187, jul./ dez. 2007 empíricas, por permitir ao adolescente a ampliação da consciência sobre si, sobre o mundo do trabalho e sobre o lazer.

\section{Aprimoramentos técnicos do BBT}

Ao estudar as possibilidades informativas do BBT no processo de clarificação dos interesses ocupacionais, Melo-Silva e Jacquemin $(1997,2000)$ enfatizaram a contribuição do procedimento complementar de elaboração da história das cinco fotos preferidas desse instrumento projetivo para se avaliar efeitos de processos de orientação profissional/vocacional. Avaliaram dez estudantes, seis do sexo feminino e quatro do sexo masculino, de 15 a 21 anos de idade, matriculados no ensino médio de Ribeirão Preto, de nível social médio. Esses adolescentes foram submetidos a entrevista clínica e aplicação individual do BBT em dois momentos da orientação profissional/vocacional: no meio do processo e ao final do mesmo. As histórias das cinco fotos preferidas do BBT foram analisadas em razão da coerência interna, do engajamento profissional apontado nas elaborações dos adolescentes, bem como em virtude das incertezas e dúvidas relacionadas ao futuro profissional, comparando-se as histórias elaboradas nos dois momentos (no meio e ao final) do processo de orientação profissional/vocacional. Os autores concluíram que as histórias das cinco fotos preferidas do BBT ampliaram as possibilidades diagnósticas dessa técnica projetiva, pois permitiram seguir a evolução da tomada de consciência do adolescente durante a orientação, evidenciando o processo de elaboração dos conflitos internos e externos que se apresentam ao sujeito, de forma a favorecer a visualização de possíveis soluções.

Ainda almejando o incremento do BBT como recurso técnico nos processos de orientação profissional/ vocacional, Melo-Silva, Assoni e Bonfim (2001) também analisaram as histórias das cinco fotos preferidas dessa técnica, com objetivo de verificar a efetividade desse procedimento no diagnóstico e no prognóstico da resolução de conflitos vivenciados durante o processo de escolha profissional. Dessa forma, 80 adolescentes foram avaliados com o BBT, sendo 23 do sexo masculino e 37 do sexo feminino, com idade entre 15 e 20 anos. Nesse estudo, as autoras restringiram-se à análise do conteúdo das histórias das cinco fotos preferidas, tendo como base as seguintes categorias: protagonista, temporalidade, conflito profissional, desfecho e título. Foram avaliadas 160 histórias, duas de cada orientando, sendo a primeira elaborada durante o processo de orientação profissional e a segunda ao término do atendimento. Os resultados apontaram que esse novo modelo de análise das histórias das fotos preferenciais do BBT possibilitou maior clarificação e compreensão do conflito envolvido na escolha profissional dos adolescentes. Dessa forma, a 
primeira história mostrou-se mais indicativa de possibilidades diagnósticas das angústias e das dúvidas dos orientandos, enquanto a segunda história conseguiu apontar mudanças individuais nessas vivências individuais, ocorridas durante o processo de orientação profissional/vocacional. Concluíram, assim, pela indicação desse procedimento analítico da história das cinco fotos preferidas do BBT aplicada em dois momentos do processo terapêutico como uma estratégia útil e clinicamente válida para sinalizar a resolutividade ou não do processo de intervenção realizado com adolescentes em momento de escolha ocupacional.

Sempre em busca do aprimoramento técnico do Teste de Fotos de Profissões na realidade nacional, Noce (2003) defendeu seu mestrado apresentando as possibilidades de composição de uma versão reduzida da forma masculina do BBT-Br. Nesse mesmo trabalho apresentou também parâmetros normativos para essa nova forma da técnica para estudantes do ensino médio de Ribeirão Preto. A questão básica norteadora desse estudo é a existência, no conjunto de fotos do BBT original, de maior número de estímulos representativos dos fatores $\mathrm{S}$, $\mathrm{V}, \mathrm{G}$ e Z (16 fotos), enquanto os outros quatro fatores possuem apenas oito representações no teste. Procurou-se, entre outras justificativas e embasamentos teórico-técnicos, igualar o número de representações desses fatores no teste, eliminando a necessidade de ponderamento final das estruturas primárias de interesse (positivas e negativas). Essa primeira etapa envolveu a análise de conteúdo das 32 fotos do BBT original, representativas dos fatores S, V, G e $Z$, por psicólogos experientes nesse instrumento projetivo, solicitando que emitissem parecer justificado sobre o poder representativo de cada uma delas a respeito de seu respectivo fator Achtnich (análise de conteúdo das fotos). Entremeando essa análise a outros indicadores técnicos da própria técnica, a pesquisadora chegou a uma forma reduzida do BBT-Br masculino, composta por 64 fotos, estando agora cada fator motivacional de Achtnich representado por oito fotos no teste. $\mathrm{Na}$ segunda etapa de seu estudo, aplicou a forma reduzida do BBT-Br (masculino) em 345 adolescentes do sexo masculino do ensino médio de Ribeirão Preto, sendo 174 alunos provenientes de escolas particulares e 171 de escolas públicas, elaborando padrões normativos para essa nova versão do instrumento. Além disso, testou o poder informativo da forma original do BBT-Br com sua versão reduzida em outro grupo de adolescentes, submetidos às duas formas da técnica. Seus resultados apontaram existir equivalência informativa entre a versão masculina completa do BBT-Br (96 fotos) e sua versão reduzida (64 fotos), disponibilizando, dessa forma, nova alternativa de aplicação desse instrumento projetivo de clarificação de interesses em nossa realidade sociocultural contemporânea.

\section{O BBT em recursos humanos}

Almejando verificar suas possibilidades informativas em contextos de seleção e de treinamento de pessoal, Welter (1998b) apresentou sua experiência de utilização do Teste de Fotos de Profissões (BBT) na clarificação do perfil de inclinação profissional de candidatos a cargos de nível gerencial em uma indústria automobilística. Para identificar os candidatos com perfil compatível com a exigência profissional de cada função, a autora avaliou 21 candidatos do sexo masculino, com idade média de 34 anos e formação profissional em diversas modalidades da engenharia. A partir dos resultados obtidos, observou-se elevado número de escolhas positivas, baixo número de rejeições e escolhas indiferentes dentro da média. $\mathrm{O}$ fator mais escolhido foi o $\mathrm{V}$, seguido pelos fatores $\mathrm{G}$ e $\mathrm{O}$, enquanto os mais rejeitados foram o $\mathrm{K}$ e S. Diante desses resultados, a autora interpreta que as preferências do grupo avaliado caracterizam-se pelo cumprimento de ordens e diretrizes externas, com as situações de risco sob controle, apontando, assim, preferência pela divisão de responsabilidades com a equipe, com discussões e troca de idéias em busca de um consenso. Esses resultados levaram a autora a concluir que o BBT possibilitou apreender o perfil de inclinação dos candidatos, permitindo a identificação de profissionais com maior concordância com o perfil de exigência profissional do cargo pretendido e, também, o encaminhamento adequado do profissional, segundo seu perfil, à melhor função correspondente. Dessa forma, a autora confirma a relevância e a riqueza das informações obtidas por meio dessa técnica projetiva para os processos de seleção de pessoal e para a área de recursos humanos, ampliando as possibilidades de aplicação da técnica no contexto nacional.

Recentemente também foi conduzido um estudo de aplicação do BBT-Br em adolescentes ingressantes em cursos profissionalizantes em São Luís (MA). Esse trabalho foi desenvolvido por Souza, Freire, Luz e Fonseca (2006) objetivando conhecer o nível motivacional de estudantes de 8. série do ensino fundamental para realizar cursos de mecânica e de eletroeletrônica, verificando sobretudo a capacidade produtiva, de atenção e de concentração com manuseio de máquinas, grau de objetividade e determinação desses adolescentes. Os autores argumentaram que o BBT-Br mostrou-se recurso técnico fundamental para o conhecimento e a avaliação dos potenciais dos estudantes, podendo-se, a partir desses resultados, elaborar programas mais específicos para seu treinamento profissionalizante.

Em seqüência a esta perspectiva de acompanhamento dos trabalhos de aplicação do BBT-Br na área de recursos humanos, Welter (2007a; 2007b) estudou a correlação dos indicadores desse instrumento projetivo com as informações obtidas por meio do Humanguide, técnica de avaliação psicológica informatizada em formato 
de inventário com indutores verbais, e por meio do $16 \mathrm{PF}$. Avaliou 87 profissionais oriundos de diversas empresas, de forma coletiva, encontrando resultados confirmadores dos sinais dessas diferentes estratégias de avaliação psicológica, apontando possibilidades de sua aplicação válida e útil em contextos de seleção de pessoal no Brasil.

\section{Considerações finais}

Consegue-se depreender dessa passagem histórica e temática, pelos trabalhos desenvolvidos com o Teste de Fotos de Profissões (BBT) no contexto brasileiro, a importância e a grande utilidade desse instrumento projetivo de clarificação profissional para os campos da orientação profissional/vocacional, seleção e treinamento de pessoal, bem como para recolocações ocupacionais. Por suas adequadas características técnicas e constante investimento de investigações científicas em nossa realidade sociocultural, considera-se que o BBT, agora BBT-Br, tem um caminho bastante promissor entre os recursos profissionais dos psicólogos, otimizando possibilidades interventivas com diferentes grupos de indivíduos, fato este também reconhecido por Noce (2007) e Pasian (2007).

Pode-se refletir, nessas passagens anteriores, sobre a questão da escolha de uma carreira, seja para um adolescente seja para um adulto em reorientação seja para um candidato em processo de seleção. Estes momentos tendem a ser vivenciados com muitas dúvidas e sentimentos angustiantes. Para Achtnich (1991), é esperada do candidato uma conduta ativa em seu processo de clarificação profissional, no qual o orientador atuará como avaliador de suas capacidades (inteligência, dons, habilidades específicas, personalidade) juntamente com a estrutura de inclinação da profissão pretendida, inserida no mundo do trabalho. As chances de sucesso e realização profissional dependem da concordância entre as capacidades do candidato e as inclinações exigidas na profissão a se escolher. Para aqueles que buscam a ajuda profissional, esses fatores nem sempre são conscientes ou esclarecidos. Para isso, o BBT se mostra como um instrumento bastante útil na clarificação, tanto das profissões como das motivações e inclinações do candidato.

Nas palavras do autor do BBT:

[...] o rendimento profissional depende diretamente da satisfação e do interesse que se dedica ao trabalho e, assim, a clarificação das inclinações toma uma significação prognóstica. Quando a profissão escolbida não corresponde às inclinaçôes falamos de escolha profissional discordante, a qual ocasiona uma insatisfação e, por conseguinte, uma queda do rendimento. No melhor dos casos, uma mudança de emprego pode ocorrer; no pior, a escolh a discordante pode provocar a doença $e$ as reaçôes psíquicas mórbidas. (Achtnich, 1991, p. 11)
Tendo por base essas possibilidades e a partir dos trabalhos anteriormente apresentados, desenvolvidos em diversos centros de pesquisa nacionais, pode-se depreender que o BBT elaborado por Achnitch configura-se como um instrumento de avaliação psicológica útil, válido e padronizado para o contexto sociocultural brasileiro. As pesquisas realizadas na realidade de nosso país estudaram tanto seus aspectos técnicos quanto suas possibilidades de aplicação em diferentes contextos, como já apontou Noce (2003). Diante dessas evidências, portanto, reiteram-se os argumentos estimuladores do uso do BBT-Br no contexto brasileiro, não apenas na área de orientação profissional/ vocacional de adolescentes, mas também como recurso avaliativo promissor na área de seleção e recrutamento de pessoal e nas recolocações profissionais. Além disso, poderia ser útil como recurso avaliativo na investigação de sucessos e insucessos ocupacionais ou para se compreender processos de adoecimento associados a questões do exercício profissional, como postulava inicialmente o próprio autor desse instrumento projetivo (Achtnich, 1991).

A fim de ampliar e aprimorar a produção do conhecimento sobre o BBT em diferentes contextos e cenários, novas investigações podem ser sugeridas na linha de estudos longitudinais e transculturais. Além disso, avaliar eventual efeito da dinâmica familiar nas inclinações motivacionais, bem como estudar o perfil de interesses de grupos profissionais específicos e tentar avaliar possíveis motivos de mudanças de profissão, configuram-se como linhas investigativas profícuas para esse instrumento projetivo. Por fim, ainda cabe destacar a importância do uso do BBT em contextos clínicos, bem como sua aplicação na análise das necessidades em atividades de lazer, entre outras possibilidades, de forma a otimizar recursos técnicos de promoção da saúde mental.

\section{Referências}

Abade, F. L. (2005). Orientação profissional no Brasil: uma revisão histórica da produção científica. Revista Brasileira de Orientação Profissional, 6(1), 15-24.

Achtnich, M. (1971). Berufsbilder-Test: versão masculina. Bern: Hans Huber.

Achtnich, M. (1973). Berufsbilder-Test: versão feminina. Bern: Hans Huber.

Achtnich, M. (1979). Der Berufsbilder Test: Projektives Verfahren zur Abkläerung der Berufsneigung. Bern: Hans Huber.

Achtnich, M. (1988). Introduction au Test de Photos de Professions (BBT) et a son utilisation dans la consultation d'orientation professionnelle et de carrière. Revue de Psychologie Appliquée, 38(4), 295-324. 
Achtnich, M. (1991). O BBT - Teste de Fotos de Profissões: método projetivo para a clarificação da inclinação profissional. (J. Ferreira Filho, Trad.). São Paulo, SP: Centro Editor de Testes e Pesquisas em Psicologia.

Barros, D. T. (2005). Avaliação psicológica em orientação profissional. Resumos do VII Encontro Mineiro de Avaliação Psicológica (EMAP). Cem anos de criação de testes psicológicos: teorização e prática, Belo Horizonte, 10.

Bernardes, E. M. (2000). O Teste de Fotos de Profissões (BBT) de Achtnich: um estudo longitudinal com adolescentes. Dissertação de Mestrado. Ribeirão Preto: Universidade de São Paulo - Faculdade de Filosofia, Ciências e Letras de Ribeirão Preto.

Bernardes, E. M. \& Jacquemin, A. (2002). O Teste de Fotos de Profissões (BBT) de Achtnich: um estudo longitudinal com adolescentes. São Paulo, SP: Vetor.

Boudrenghien, G. (1983). Contribution a l'utilisation du Berufsbilder-Test de M. Achtnich. Bulletin de Psychologie Scolaire et d'Orientation, 32(2), 49-61.

Campos, M. I. R. S. (2003). BBT-Br na avaliação das relações entre o perfil vocacional da profissão e a saúde. Dissertação de Mestrado. Itatiba: Universidade São Francisco.

Chaghaghi, F. \& Jobin, C. (1983). L'application du test BBT (Berufsbildertest) à une population de Suisse Romande. Orientation et Formation Professionnelles, 68(6), 43-51.

Conselho Federal de Psicologia (Brasil) (2003). Edital CFP $\mathrm{n}^{\mathrm{o}} 1$, de 17 de julho de 2003. Processo de avaliação dos testes psicológicos. Jornal do Conselho Federal de Psicologia, 18(75).

De Ketele, R. (1982). Le Berufsbilder-Test (BBT) de Martin Achtnich dans la consultation d'orientation: instrument de mesure ou support d'une demarche? Bulletin de Psychologie Scolaire et d'Orientation, 31(4), 14-25.

Draime, J. \& Jacquemin, A. (1989). Os testes em orientação vocacional e profissional. Arquivos Brasileiros de Psicologia, 41(3), 95-99.

Foulon, R. (1981). Contribuition à la validation du BerufsbilderTest de Martin Achtnich. (Memorial não publicado). Louvain-la Neuve, Belgique: Université Catholique de Louvain - Faculté de Psychologie et des Sciences de l'Education.

Guelli, A. V. \& Jacquemin, A. (1997). Etude de l'efficacité de l'utilisation du BBT - Test de Photos de Professions et de la Technique R-O sur certains processus psychologiques impliqués dans le choix profissionnel. Resumos da International Conference of International Association for Educational and Vocational Guidance, Brasov, Romênia, 12.
Jacquemin, A. (1982). Novas perspectivas em orientação vocacional e profissional. Arquivos Brasileiros de Psicologia, 34(4), 127-132.

Jacquemin, A. (1985). O Berufsbilder-Test (BBT) de Achtnich: uma nova perspectiva em orientação vocacional e profissional. Boletim de Psiquiatria, 18(1/2), 10 .

Jacquemin, A. (1989). L'etat des recherches sur le BBT au Brésil. Bulletin de Psychologie Scolaire e d'Orientation, 41(3), 95-99.

Jacquemin, A. (1995). Le BBT chez les étudiants brésiliens: quelques resultants préliminaires. Anais da International Conference of International Association for Educational and Vocational Guidance, Lisboa, Portugal, 307-312.

Jacquemin, A. (1997). Le Test de Photos de Professions de Martin Achtnich: adaptation au contexte socio-culturel brésilien. Resumos da International Conference of International Association for Educational and Vocational Guidance, Brasov: Romênia, 13.

Jacquemin, A. (1999). Le Test de Photos de Professions de M. Achtnich: adaptation au contexte socio-culturel brésilien (BBT-Br). Resumos do XVI International Congress of Rorschach and Projective Methods, Amsterdam, Holanda, 323.

Jacquemin, A. (2000). O BBT-Br: Teste de Fotos de Profissões: normas, adaptação brasileira, estudos de caso. São Paulo, SP: Centro Editor de Testes e Pesquisas em Psicologia.

Jacquemin, A. \& Nunes, S. L. (1985). O Berufsbilder-Test (BBT) de Achtnich. Problema de validade interna II. Boletim de Psiquiatria, 18(1/2), 11-12.

Jacquemin, A. \& Nunes, S. L. (1987). Le Test d'Achtnich: un exemple d'application clinique. Rorschachiana, XVI, 243-244.

Jacquemin, A. \& Nunes, S. L. (1990). Le Berufsbilder-Test de Achtnich chez des étudiants brésiliennes. Resumos da XIII International Congress of Rorschach and Projective Techniques, Paris, França, 232.

Jacquemin, A. \& Pasian, S. R. (1991). O BBT no Brasil. Em M. Achtnich. O BBT, Teste de Fotos de Profissões: método projetivo para a clarificação da inclinação profissional (pp. 208-222). São Paulo: Centro Editor de Testes e Pesquisas em Psicologia.

Jacquemin, A. \& Nunes, S. L. (1995). Le BBT chez les étudiants brésiliennes: forme masculine ou forme féminine? Anais da International Conference of International Association for Educational and Vocational Guidance, Lisboa, Portugal: Soc. Astória, 313-317.

Jacquemin, A., Melo-Silva, L. L. \& Pasian, S. R. (2002). O Berufsbilder Test (BBT) - Teste de Fotos de Profissões 
em Processos de Orientação Profissional. Em R. S. Levenfus \& D. H. P. Soares (Orgs.). Orientação vocacional ocupacional: novos achados teóricos, técnicos e instrumentais para a clínica, a escola e a empresa (pp. 247-261). Porto Alegre: ArtMed.

Jacquemin, A., Xavier, M. A., Wierman, M. L. \& Lima, M. J. (1985). Problema da validade interna I. Boletim de Psiquiatria, 18(1/2), 11.

Jacquemin, A., Vendruscolo, J., Okino, E. T. K., Barboni, F. D., Santos, C. R. O., Motta, A. M. A. \& Salles, C. F. (1995). O Berufsbilder Test (BBT) de Martin Achtnich. Adaptação para o contexto cultural brasileiro. Anais do I Encontro da Sociedade Brasileira de Rorschach e outros métodos projetivos, Ribeirão Preto, SP: VGA, 237-248.

Jacquemin, A., Noce, M. A., Santos, C. O., Marcos, M., Assoni, R. F. \& Bianchi, S. H. (1998). O Berufsbilder Test (BBT) de Martin Achtnich: adaptação para o contexto cultural brasileiro. Relatório final. Ribeirão Preto: Universidade de São Paulo - Faculdade de Filosofia, Ciências e Letras de Ribeirão Preto.

Jacquemin, A., Noce, M. A., Assoni, R. F., Okino, E. T. K., Prado, A. P. B. A. \& Zeoti, D. M. (2000). O Teste de Fotos de Profissões (BBT) no contexto brasileiro: estudo da validade interna da forma feminina. Anais do II Congresso Nacional da Sociedade Brasileira de Rorschach e outros métodos projetivos, Porto Alegre: AGE, 251-257.

Jacquemin, A., Noce, M. A., Assoni, R. F., Okino, E. T. K., Kawakami, E. A. \& Pasian, S. R. (2001). O Berufsbilder Test (BBT) de Achtnich: adaptação brasileira da forma feminina. Resumos do IV Encontro da Sociedade Brasileira de Rorschach e Outros Métodos Projetivos, Ribeirão Preto, 130.

Jacquemin, A., Pasian, S. R., Assoni, R. F., Okino, E. T. K., Corlatti, C. T., Kawakami, E. A., Frazatto, L. \& Jardim, M. L. C. (2002). O Berufsbilder-Test (BBT) de Martin Achtnich: adaptação da forma feminina para o contexto cultural brasileiro. Relatório de Pesquisa. Ribeirão Preto: Universidade de São Paulo - Faculdade de Filosofia, Ciências e Letras de Ribeirão Preto.

Jacquemin, A., Pasian, S. R., Assoni, R. F., Okino, E. T. K., Corlatti, C. T., Kawakami, E. A., Frazatto, L. \& Jardim, M. L. C. (2003). O Berufsbilder Test (BBT) de Martin Achtnich: adaptação da forma feminina para o contexto cultural brasileiro. Relatório de Pesquisa. Ribeirão Preto: Universidade de São Paulo - Faculdade de Filosofia, Ciências e Letras de Ribeirão Preto.

Jacquemin, A., Okino, E. T. K., Noce, M. A., Assoni, R. F. \& Pasian, S. R. (2006). O BBT-Br Feminino. Teste de Fotos de Profissões: adaptação brasileira, normas e estudos de caso. São
Paulo, SP: Centro Editor de Testes e Pesquisas em Psicologia.

Jardim-Maran, M. L. C. (2004). Dinamismo psicológico na adolescência diante da escolha profissional: uma contribuição do $B B T-B r$ e do Questionário Desiderativo. Dissertação de Mestrado. Ribeirão Preto: Universidade de São Paulo Faculdade de Filosofia, Ciências e Letras de Ribeirão Preto.

Jardim-Maran, M. L. C. (2007). A escolha profissional de adolescentes segundo o BBT-Br e o Questionário Desiderativo. Resumos do III Congresso Brasileiro de Avaliação Psicológica e XII Conferência Internacional de Avaliação Psicológica: Formas e Contextos, [CD], João Pessoa, 102.

Leitão, L. B. C. O. G. M. (1984). Reflexões sobre um teste projectivo de orientação vocacional: O "Berufsbilder-Test". Trabalho-síntese com vistas às provas de aptidão pedagógica e capacidade científica para passagem da categoria de assistente-estagiária à de assistente em psicologia. Coimbra, Portugal: Universidade de Coimbra - Faculdade de Psicologia e Ciências da Educação.

Leitão, L. B. C. O. G. M. (1993). Contributos para um modelo de avaliação dinâmica dos interesses. Tese de Doutorado. Coimbra, Portugal: Universidade de Coimbra Faculdade de Psicologia e Ciências da Educação.

Leleux, A. L. (1982). Le Berufsbilder Test (BBT) de Martin Achtnich: présentation du test. Bulletin de Psychologie Scolaire et d'Orientation, 31(4), 1-13.

Lievyns, C. (1987). Le Berufsbilder-Test de M. Achtnich: tentative de standardization d'une population belge âgée de 17 ans 6 moins à moins de 24 ans. Mémoire Licenciée en Psychologie. Louvain-la-Neuve, Belgique: Université Catholique de Louvain - Faculté de Psychologie et des Sciences de l'Education.

Marchand, B. (1983). Le test visuel d'intérêts et de personnalité (BBT) en Suisse Romande. Orientation et Formation Professinnnelles, 68(6), 41-42.

Melo-Silva, L. L. (2000). Intervenção em orientação vocacional/ profissional: avaliando resultados e processos. Tese de Doutorado. Ribeirão Preto: Universidade de São Paulo - Faculdade de Filosofia, Ciências e Letras de Ribeirão Preto.

Melo-Silva, L. L. \& Jacquemin, A. (1997a). L'histoire des cinq photos préférées au BBT à deux momentes du processus d'orientation professionelle. Reviste de Pedagogie, 1(12), 65-69.

Melo-Silva, L. L. \& Santos, M. A. (1997b). O BBT como instrumento diagnóstico em orientação profissional e 
psicoterapia. Resumos da XXVII Reunião Anual de Psicologia, Ribeirão Preto, 75-76.

Melo-Silva, L. L. \& Santos, M. A. (1998). O BBT como instrumento diagnóstico em orientação profissional: uma abordagem psicodinâmica. Revista da Associação Brasileira de Orientadores Profissionais, 2(1), 59-76.

Melo-Silva, L. L. \& Jacquemin, A. (2000). Contribuição para a interpretação do BBT de Martin Achtnich: a história das cinco fotos preferidas. Psic: Revista de Psicologia da Vetor Editora, 1(3), 72-79.

Melo-Silva, L. L. \& Jacquemin, A. (2001a). BBT - Teste de Fotos de Profissões, método projetivo para a inclinação profissional de Martin Achtnich. Em D. H. P. Soares (Org.). Anais do IV Simpósio Brasileiro de Orientação Vocacional \& Ocupacional. I Encontro de Orientadores Profissionais do Mercosul, São Paulo: Vetor, 301-311.

Melo-Silva, L. L. \& Jacquemin, A. (2001b). Intervenção em orientação vocacionall profissional: avaliando resultados $e$ processos. São Paulo, SP: Vetor.

Melo-Silva, L. L. \& Noce, M. A. (2004). O Teste de Fotos de Profissões (BBT) enquanto método projetivo em orientação profissional: estudos de caso. Em Z. B. Vasconcelos \& I. D. Oliveira (Orgs.). Orientação vocacional: alguns aspectos teóricos, técnicos e práticos (pp. 141186). São Paulo: Vetor.

Melo-Silva, L. L., Noce, M. A. \& Andrade, P. P. (1999). A estrutura de inclinação profissional em adolescentes. Em Associação Brasileira de Orientação Profissional. Resumos do IV Simpósio Brasileiro de Orientação Vocacional \&o Ocupacional/ I Encontro de Orientadores Profissionais do Mercosul, Florianópolis, SC, 28.

Melo-Silva, L. L., Assoni, R. F. \& Bonfim, T. (2001). A história das cinco fotos preferidas do BBT: proposta de um modelo de análise. Em D. H. P. Soares (Org.). Anais do IV Simpósio Brasileiro de Orientação Vocacional \& Ocupacional. I Encontro de Orientadores Profissionais do Mercosul, São Paulo: Vetor, 171-181.

Melo-Silva, L. L., Noce, M. A., Andrade, P. P. (2003). Interesses em adolescentes que procuram orientação profissional. Psic: Revista de Psicologia da Vetor Editora, $4(2), 6-17$.

Noce, M. A. (2003). O BBT-Br-Teste de Fotos de Profissões: proposta de versão reduzida da forma masculina e seus padrões normativos. Dissertação de Mestrado. Ribeirão Preto: Universidade de São Paulo - Faculdade de Filosofia, Ciências e Letras de Ribeirão Preto.

Noce, M. A. (2007). Diversidade de investigações sobre o Teste de Fotos de Profissões (BBT-Br) no contexto brasileiro. Resumos do III Congresso Brasileiro de Avaliação
Psicológica e XII Conferência Internacional de Avaliação Psicológica: Formas e Contextos, [CD], João Pessoa, 101.

Nunes, S. L. (1989). O Berufsbilder Test de Achtnich: O problema da validade interna. Dissertação de Mestrado. São Paulo: Universidade de São Paulo - Instituto de Psicologia.

Okino, E. T. K., Noce, M. A., Assoni, R. F., Corlatti, C. T., Pasian, S. R. \& Jacquemin, A. (2003). A adaptação do BBT - Teste de Fotos de Profissões - para o contexto sociocultural brasileiro. Revista Brasileira de Orientação Profissional, 4(1/2), 87-96.

Pasian, S. R. (2007). Possibilidades do Teste de Fotos de Profissões (BBT-Br) em processos de orientação profissional. Resumos do III Congresso Brasileiro de Avaliação Psicológica e XII Conferência Internacional de Avaliação Psicológica: Formas e Contextos, [CD], João Pessoa, 157158.

Ribeiro, A. V. G. (1998). Algumas contribuições para o uso dos métodos BBT e R-O em orientação profissional. Dissertação de Mestrado. Ribeirão Preto: Universidade de São Paulo Faculdade de Filosofia, Ciências e Letras de Ribeirão Preto.

Savickas, M. L. (2004). Um modelo para avaliação de carreira. Em L. M. Leitão (Org.). Avaliação psicológica em orientação escolar e profissional (pp. 21-42). Coimbra: Quarteto.

Sbardelini, E. T. B. (1997). A reopção de curso na Universidade Federal do Paraná. Tese de Doutorado. Ribeirão Preto: Universidade de São Paulo - Faculdade de Medicina de Ribeirão Preto.

Souza, T. F., Freire, C. C. R., Luz, M. L. \& Fonseca, S. A. (2006). A utilização do BBT-Br como instrumento de seleção de adolescentes. Resumos do IV Congresso Nacional da Associação Brasileira de Rorschach e métodos projetivos, Brasilia, 221.

Szondi, L. (1970). Tratado del diagnostico experimental de los instintos. Traducción de la segunda edición alemana. Madrid: Biblioteca Nueva.

Walgraffe, R. V. B. (1989). Une pratique clinique de l'orientation. Bulletin de Psychologie, 38(1), 103-111.

Welter, G. M. R. (1998a). Estudo de correlação com o BBT - Teste de Fotos de Profissões: aplicação coletiva com a série masculina e aplicação individual com a série feminina no mesmo sujeito. Anais do III Encontro da Sociedade Brasileira de Rorschach e Outros Métodos Projetivos, Ribeirão Preto, 174-179.

Welter, G. M. R. (1998b). Uma experiência com o BBT Teste de Fotos de Profissões em recursos humanos de uma indústria automobilística. Anais do III Encontro da 
Sociedade Brasileira de Rorschach e Outros Métodos Projetivos, Ribeirão Preto, 180-184.

Welter, G. M. R. (2007a). Humanguide: evidências de validade da versão brasileira. Dissertação de Mestrado. Itatiba: Universidade São Francisco.

Welter, G. M. R. (2007b). Estudo de correlação entre o Humanguide e o BBT - Teste de Fotos de Profissões. Resumos do I Congresso Latinoamericano de Orientação
Profissional da ABOP e VIII Simpósio Brasileiro de Orientação Vocacional \& Ocupacional, Bento Gonçalves, 137.

Recebido em fevereiro de 2007 Reformulado em agosto de 2007 Aprovado em setembro de 2007

Sobre as autoras:

Sonia Regina Pasian é psicóloga, mestre em Filosofia (UFSCar), doutora em Saúde Mental (FMRP-USP), docente do Departamento de Psicologia e Educação (pós-graduação e graduação) da Faculdade de Filosofia, Ciências e Letras de Ribeirão Preto da Universidade de São Paulo, onde coordena o Centro de Pesquisas em Psicodiagnóstico. É assessora científica de periódicos e agências de pesquisa e atual presidente da Associação Brasileira de Rorschach e Métodos Projetivos (ASBRo).

Erika Tiemi Kato Okino é psicóloga do Departamento de Psicologia e Educação da Faculdade de Filosofia, Ciências e Letras de Ribeirão Preto, da Universidade de São Paulo, exercendo função de apoio ao ensino e à pesquisa. É mestre em Psicologia pelo Programa de Pós-Graduação em Psicologia da FFCLRP/USP, onde atualmente desenvolve doutorado na área de Avaliação Psicológica, voltado à análise das evidências de validade do BBT-Br.

Lucy Leal Melo-Silva é psicóloga, doutora em Psicologia pela Universidade de São Paulo (USP), docente do Programa de Pós-Graduação em Psicologia e do curso de Psicologia do Departamento de Psicologia e Educação, da Faculdade de Filosofia, Ciências e Letras de Ribeirão Preto (FFCLRP-USP). É também editora da Revista Brasileira de Orientação Profissional e autora de livros na área da Orientação Profissional e Formação em Psicologia. 
